\title{
Analyse au stade juvénile d'un test multilocal de clones d'épicéa commun (Picea abies (L.) Karst.). Variabilité génétique
}

\author{
H. van de Sype et B. Roman-Amat
}

INRA, Station d'amélioration des arbres forestiers, Ardon, 45160 Olivet, France

(reçu le 30-3-1988; accepté le 20-7-1988)

Résumé - La croissance en hauteur et la tardiveté du débourrement végétatif ont été étudiées après 5 ans passés en forêt pour 1130 clones d'épicéa commun testés dans 3 sites. Les clones proviennent de 30 provenances, principalement polonaises, représentées au total par 300 descendances maternelles récoltées en forêt.

L'héritabilité clonale de la hauteur totale décroît constamment depuis la plantation jusqu'à un minimum compris entre 0,47 et 0,69 selon les sites. La pousse annuelle en plantation n'a pas encore atteint son maximum et son héritabilité augmente dès la troisième année pour atteindre 0,35 à 0,58 selon les sites. La tardiveté du débourrement en 1986 est fortement héritable $(0,79$ à 0,89$)$. Les principales sources de variabilité sont d'origine individuelle (entre ramets) ou clonale, la variabilité liée aux niveaux provenance et famille étant nettement plus faible, quoique significative.

D'un site à l'autre, les résultats concordent pour la décomposition de la variance et les valeurs d'héritabilité clonale notamment. Seule la corrélation génétique clonale entre la vigueur et le débourrement est différente selon les sites : $r=-0,12$ (pour 758 clones) et $r=+0,28$ (pour 1040 clones). Cette différence s'explique probablement par le fait que la population clonale étudiée varie d'un site à l'autre.

D'après les valeurs moyennes des clones, déterminées en analysant globalement les résultats des 3 sites, les clones sont à la fois plus tardifs ( 8 jours) et plus vigoureux $(+19$ à $29 \%$ ) que les témoins. Une attention particulière est portée aux répercussions qu'ont eues sur les caractéristiques de la population clonale les sélections préliminaires réalisées en forêt et en pépinière. Au total, les fortes variabilités génétiques et héritabilités clonales pour la vigueur et le débourrement, ainsi que l'absence ou la faiblesse de la corrélation entre ces deux caractères au niveau clonal, suggèrent qu'une sélection efficace pourra être entreprise.

Picea abies - croissance - débourrement végétatif - variance génétique - héritabilité - corrélation génétique - clone

Summary - Analysis at the juvenile stage of a multilocate clonal test of Norway spruce (Picea abies (L.) Karst.). Genetic varlability. A study of height increment and flushing was performed with 1130 clones of Picea abies after 5 years in the field. These clones were the result of a selection in nursery at age 4, from polish provenances and progenies. Material was distributed in 3 localities.

For total height, the clonal mean was greater than reference provenances. Clonal heritability showed a decline during the first year after planting. After adjustment to block effect, the cumulated variance for provenance, progeny and clone levels, termed genetic variance, represented 17-28\% of the total variance. For annual shoot increment, the same feature was observed with absolute 
values. Nevertheless, evolution of clonal heritability differed, and seemed to increase after the second year. Cumulated shoot increment from planting to the 5th year was $20 \mathrm{~cm}$ higher for clones than for reference provenances. Heritabilities were $0.32,0.56$ and 0.63 respectively for the 3 localities, and genetic variance represented 13-22\% of the total variance. Flushing, studied on sites 2 and 3 during the spring of 1986, exhibited a clonal heritability between 0.79-0.89, and a genetic variance proportion varying from $52-57 \%$.

Genetic correlations at clone level between height and flushing were -0.12 (site 2, 758 clones) and +0.28 (site 3,1040 clones); this change may have been caused by a difference in genetic composition from one site to another. Correlations of clonal means on 2 sites were significant. After adjustment to site effect, clones were better than references for height ( +19 to $29 \%)$ and flushing (8 days). Overall clonal heritabilities were high : 0.86 on flushing, 0.81 on total height and 0.62 on cumulated shoot increment in plantation.

In conclusion, three main points are discussed. (i) The reduction with time of the total height clonal heritability may reflect nursery effect, or express changes in genetic regulation of growth from nursery to plantation sites. (ii) The preliminary selection steps in forest and in nursery which led to the selection of these clones did not erase the high natural variability due to provenance and progeny-within-provenance levels. (iii) For the following selection steps, results point to the cumulated shoot increment in plantation as the best criterion for vigor description. Finally, the clonal population studied seems to be suitable for further selection, and may ultimately provide a performant multiclonal variety.

Picea abies - growth - flushing - genetic variance - heritability - genetic correlation - clone

\section{Introduction}

Dans la stratégie d'amélioration génétique de l'épicéa commun, la voie clonale paraît une méthode très intéressante. Par rapport à la voie sexuée, la multiplication végétative facilite tout d'abord une sortie rapide de matériel sélectionné, l'étape de la fructification étant supprimée (Kleinschmit, 1983). Elle facilite la gestion du matériel génétique en offrant la possibilité d'utiliser ensemble des populations distinctes (Kleinschmit, 1983). Elle entraîne également un gain génétique important en fixant une partie de la variabilité individuelle et en valorisant à la fois les variabilités additive et non additive (Matheson et Lindgren, 1985; Burdon, 1986; Roulund et al., 1986). Enfin, de par sa rapidité de mise en œuvre, elle favorise la création de variétés adaptées à des conditions particulières (Kleinschmit, 1983; Burdon, 1986).

La voie clonale présente aussi quelques risques. La crainte d'une dérive des qualités du matériel par vieillissement progres- sif des clones au cours des cycles de propagation serait négligeable (Kleinschmit, 1983; St Clair et al., 1985). Les résultats expérimentaux en ce domaine sont toutefois relativement récents chez l'épicéa. En revanche, le risque le plus important semble être une forte réduction de la base génétique. Ceci entraînerait l'instabilité des clones constituant les variétés multiclonales diffusées pour le reboisement et, par voie de conséquence, une réduction de la plasticité vis-à-vis des biotopes, parasites ou accidents climatiques (Kleinschmit, 1983; Burdon, 1986). Ainsi, l'instabilité des clones dans les différents environnements serait élevée (Burdon, 1986; St Clair et Kleinschmit, 1986; Huehn et al., 1987).

En France, la méthode clonale, appliquée à l'épicéa commun, a débuté par des tests de provenance qui ont montré la supériorité de certaines origines polonaises (Lacaze et Polge, 1970) pour des caractères économiquement importants comme la hauteur ou la tardiveté du 
débourrement végétatif (afin de limiter les risques liés aux gelées tardives de printemps). Par la suite, les semis (ou ortets) les plus performants de ces meilleures provenances et descendances ont été sélectionnés en pépinière pour ces mêmes caractères puis clonés par bouturage.

Les ramets, issus de boutures, ont été installés en forêt dans 3 tests comparatifs depuis 5 ans maintenant. Une première étude semble donc possible pour juger les clones.

Dans un premier temps, la variance phénotypique des caractères observés sera décomposée site par site selon les 3 niveaux : provenance, descendance et clone. L'évolution au cours du temps de la part relative de la variance entre clones dans la variance phénotypique guidera la recherche d'un critère de sélection juvénile pour la croissance.

L'analyse de l'interaction génotype/environnement et la méthode de sélection des clones testés seront abordées dans un article ultérieur.

\section{Matériel et Méthodes}

Matériel végétal

Le matériel végétal est issu principalement des peuplements polonais, connus pour leurs bonnes performances, tant pour la vigueur, zone des Beskides au sud, que pour la tardive-

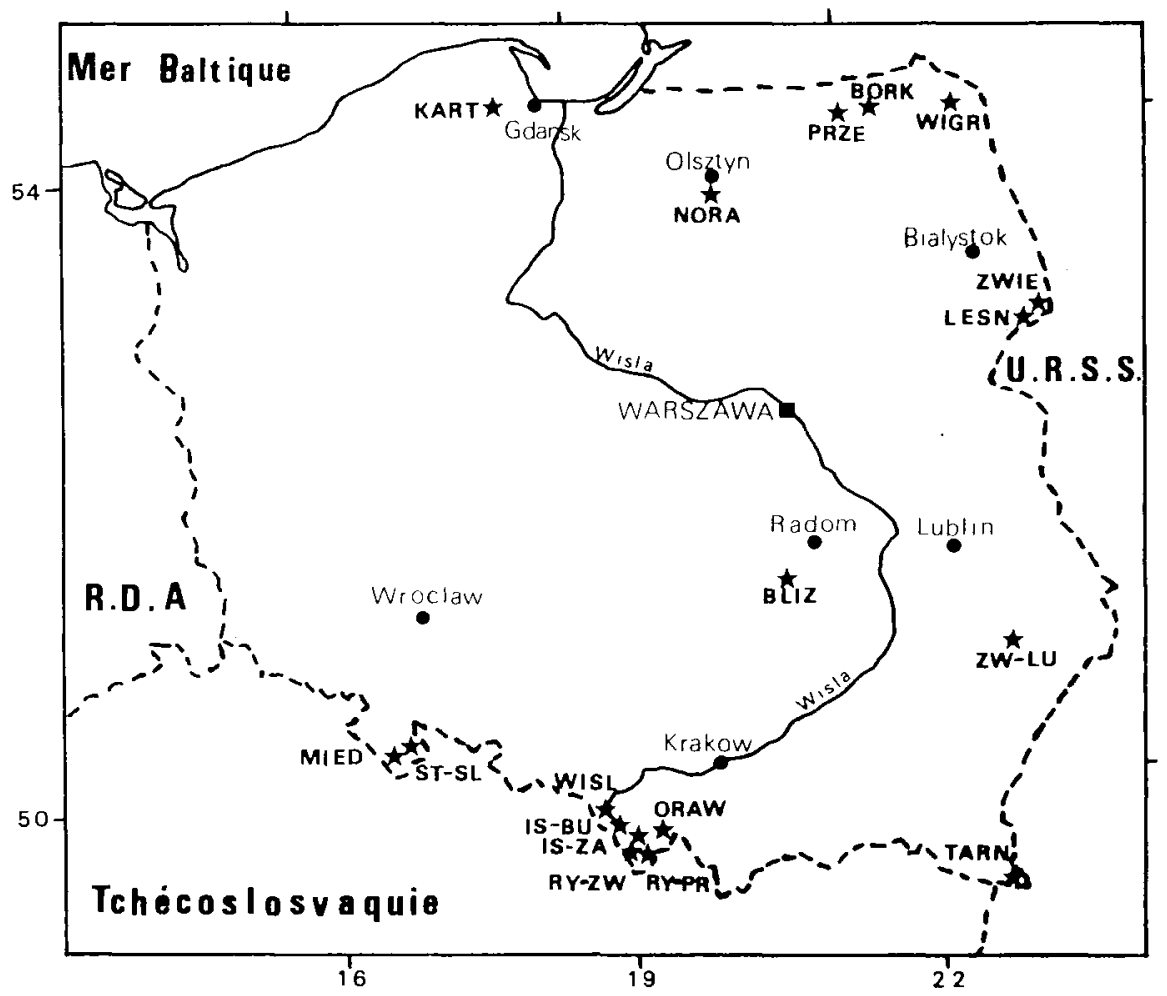

Fig. 1. Localisation des provenances polonaises. 
Tableau I. Liste du matériel étudié.

\begin{tabular}{|c|c|c|c|c|c|c|c|}
\hline \multirow[t]{3}{*}{ Provenance } & & & \multicolumn{2}{|c|}{$\begin{array}{l}\text { Nombre de } \\
\text { descendance }\end{array}$} & \multicolumn{3}{|c|}{ Clones issus de } \\
\hline & & & & & \multirow[t]{2}{*}{ Provenance } & \multicolumn{2}{|c|}{ Descendance } \\
\hline & & & IV & “+” & & $T V$ & «+» \\
\hline BLIZ & Blizyn & $\mathrm{C}$ & & & 2 & & \\
\hline BONE & Bonnétage & $\mathrm{F}$ & 19 & & 3 & 40 & \\
\hline BORK & Borki & $\mathrm{N}$ & 15 & & 1 & 62 & \\
\hline BORK «+» & Borki «+» & $\mathrm{N}$ & & 5 & & & 55 \\
\hline CHAP & Chapois & $\mathrm{F}$ & & & 7 & & \\
\hline GERA & Gérardmer & $\mathrm{F}$ & & & 1 & & \\
\hline IS-BU & Istebna-Bukoviec & $S$ & 15 & & 1 & 41 & \\
\hline IS-ZA & Istebna-Zapowiedz & $S$ & 22 & & 4 & 86 & \\
\hline ISTE «+» & Istebna «+» & $\mathrm{S}$ & & 11 & & & 57 \\
\hline KART & Kartuzy & $\mathrm{N}$ & 14 & & 2 & 36 & \\
\hline LESN «+» & Lesna “+» & $\mathrm{N}$ & & 3 & & & 22 \\
\hline MIED & Miedzygorze & $S$ & 12 & & & 23 & \\
\hline MONR 2 & Montriond 2 & $\mathrm{~F}$ & & & 1 & & \\
\hline NO-RA & Nowe-Ramuki & $N$ & & & 1 & & \\
\hline ORAW & Orawa & $\mathrm{S}$ & 5 & & & 12 & \\
\hline PRZE & Przerwanki & $\mathrm{N}$ & 11 & & 10 & 26 & \\
\hline RY-PR 1 & Rycerka-Praszywka 1 & $\mathrm{~S}$ & 17 & & 4 & 46 & \\
\hline RY-PR 2 & Rycerka-Praszywka 2 & $\mathrm{~S}$ & & & 1 & & \\
\hline RY-ZW & Rycerka-Zwardon & $\mathrm{S}$ & 17 & & 2 & 65 & \\
\hline RYCE «+» & Rycerka «+» & $\mathrm{S}$ & & 9 & & & 36 \\
\hline ST-SL & Stronie-Slaskie & $\mathrm{S}$ & 5 & & & 10 & \\
\hline TARN & Tarnawa & $\mathrm{S}$ & 26 & & 2 & 60 & \\
\hline WIGR & Wigry & $\mathrm{N}$ & 11 & & 1 & 26 & \\
\hline WISL & Wisla & $s$ & 9 & & 6 & 28 & \\
\hline WISL «+» & Wisla «+» & $\mathrm{S}$ & & 5 & & & 27 \\
\hline ZW-LU & Zwierzyniec-Lubelski & C & 12 & & 7 & 60 & \\
\hline ZWIE 1 & Zwierzyniec 1 & $\mathrm{~N}$ & 17 & & 2 & 54 & \\
\hline ZWIE 2 & Zwierzyniec 2 & $\mathrm{~N}$ & 14 & & 2 & 47 & \\
\hline ZWIE «+» & Zwierzyniec «+» & $\mathrm{N}$ & & 26 & & & 136 \\
\hline & non connu & & & & 15 & & \\
\hline Total & & & 241 & 59 & 75 & 722 & 333 \\
\hline
\end{tabular}

France (F) ; Pologne : Nord (N), Centre (C) ou Sud (S). Les témoins, semis des provenances Bonnétage, Gérardmer et lstebna, ne sont pas indiqués. Les nombres de descendance et de clone sont décalés sur la gauche pour le matériel tout-venant (TV) et sur la droite pour le matériel issu d'arbres «+»(«+).

té du débourrement, zone baltique au nord-est (Fig. 1). Au printemps 1970 , des graines de ces peuplements ont été semées en pépinière. Chaque peuplement pouvait être représenté par des semis de :

- provenance (mélange de mères non identifiées);

- descendance maternelle récoltée sur des arbres tout-venant;

- descendance maternelle récoltée sur des arbres «+».
Ces arbres «+" avaient été sélectionnés phénotypiquement en forêt par Lacaze (INRA) et Kociecki (IBL) dans une aire plus vaste que celle de la provenance ou des descendances tout-venant du peuplement correspondant. La sélection, en riveaux indépendants, portait tout d'abord sur la tardiveté du débourrement végétatif, puis sur la densité du bois estimée à l'aide d'un torsiomètre.

A cette collection, 4 peuplements français de performances connues ont été ajoutés à titre de 
Tableau II. Caractéristiques des tests clonaux

\begin{tabular}{|c|c|c|c|}
\hline Site & 1 & 2 & 3 \\
\hline $\begin{array}{l}\text { Région } \\
\text { Département } \\
\text { Localité }\end{array}$ & $\begin{array}{l}\text { Jura } \\
\text { Doubs } \\
\text { Eternoz }\end{array}$ & $\begin{array}{l}\text { Jura } \\
\text { Doubs } \\
\text { Gonsans }\end{array}$ & $\begin{array}{l}\text { Massif central } \\
\text { Creuse } \\
\text { Saint-Goussaud }\end{array}$ \\
\hline $\begin{array}{l}\text { Altitude } \\
\text { Profondeur sol } \\
\text { Sous-sol }\end{array}$ & $\begin{array}{l}500 \mathrm{~m} \\
10-45 \mathrm{~cm} \\
\text { calcaire }\end{array}$ & $\begin{array}{l}550 \mathrm{~m} \\
15-130 \mathrm{~cm} \\
\text { calcaire fissuré }\end{array}$ & $\begin{array}{l}530 \mathrm{~m} \\
10-15 \mathrm{~cm} \\
\text { arène granitique }\end{array}$ \\
\hline $\begin{array}{l}\text { Age des ramets } \\
\text { Plantation } \\
\text { Densité } \\
\text { Clones plantés } \\
\text { Témoins }\end{array}$ & $\begin{array}{l}4 \text { ans } \\
\text { fin } 1980 \\
2 \times 2 \mathrm{~m} \\
576 \\
\text { Gérardmer } \\
\text { Bonnétage }\end{array}$ & $\begin{array}{l}3 \text { ans } \\
\text { début } 1981 \\
2,75 \times 2 \mathrm{~m} \\
758 \\
\text { Gérardmer } \\
\text { Istebna }\end{array}$ & $\begin{array}{l}3 \text { ans } \\
\text { début } 1981 \\
3 \times 2 \mathrm{~m} \\
1040 \\
\text { Gérardmer }\end{array}$ \\
\hline
\end{tabular}

comparaison. Par facilité de langage, on appellera provenance l'ensemble des descendances ou clones issus d'une provenance, même s'il n'y en a qu'un seul. De la même façon, une descendance sera constituée par l'ensemble des clones qui en sont issus. Pour les arbres «+» d'un peuplement, on a considéré qu'ils constituaient une provenance à part entière.

L'élevage de ces 486 provenances et descendances en pépinière a été suivi par une sélection massale à 4 ans. Cette sélection, en niveaux indépendants, a porté sur les $5 \%$ d'individus les plus tardifs, puis sur les $30 \%$ d'individus les plus vigoureux. Parmi les 1280 semis (ortets) retenus, seuls 1130 ont donné un nombre suffisant de boutures enracinées. L'expérience a été réalisée sur ces 1130 clones issus de 300 descendances et originaires de 30 provenances soit 21 peuplements ou massifs forestiers (Tableau I).

La collecte des boutures, leur enracinement puis leur élevage ont été assurés à Orléans par D. Cornu au cours de 2 campagnes consécutives. Les ramets âgés de 3 ou 4 ans ont été plantés dans 3 sites dont les principales caractéristiques sont présentées dans le Tableau II. Ces terrains sont typiques des zones de reboisement en épicéa pour le Jura (sites 1 et 2 ) et le Massif central (site 3). Les témoins, issus de graines, sont constitués par 3 provenances, au sens strict du terme. Bonnétage est une provenance tardive du Jura alors que Gérardmer, orjginaire des Vosges, est plus précoce mais plus vigoureuse. Istebna est considérée en France comme très vigoureuse tout en demeurant tardive (Lacaze et Polge, 1970; Légnaté, 1986).
Les 3 dispositifs sont installés en blocs aléatoires incomplets avec 50 clones par bloc et 2 ramets par clone. Les clones sont représentés en moyenne par 3 à 7 ramets par site. Des plants, issus du semis des 3 provenances témoins, ont été incorporés dans chaque bloc. Comme l'indique le Tableau II, les clones ne sont pas présents dans tous les sites. Les clones communs, ayant des ramets vivants, sont respectivement au nombre de 282 (sites 1 et 2), 568 (sites 2 et 3), 437 (sites 1 et 3 ) et 257 pour les 3 sites.

\section{Mesures et analyses}

Les mesures portent sur la mortalité après 5 années de plantation (pourcentage d'arbres morts), les hauteurs tolales mesurées à la fin de chaque année en plantation et le débourrement au printemps 1986. Le débourrement est observé en 8 passages où sont notés l'absence ou la présence de bourgeon apical débourré. II est exprimé en nombre de jours après le premier passage. Pour le site 1 , cette mesure n'a pas été possible, et 56 clones n'ont pas pu être testés pour le débourrement.

\section{Analyse dans chaque site}

Pour chaque site, les données individuelles des ramets sont analysées selon 3 modèles. L'analyse du dispositif expérimental est réalisée d'après le modèle (1) :

$$
X_{k l n}=\mu+C_{k}+B_{l}+C B_{k l}+e_{k l n}
$$


avec les effets clone $\left(C_{k}\right.$, aléatoire) et bloc $\left(B_{l}\right.$, fixe). En l'absence d'interaction $\left(\mathrm{CB}_{k}\right)$, la valeur individuelle des ramets est ajustée à l'effet des blocs $\left(Y_{k n}=X_{k l n}-B_{1}\right)$. Les analyses ultérieures sont entreprises à partir de la valeur individuelle des ramets ajustée à l'effet des blocs. Une analyse au niveau clonal a été entreprise selon le modète (2) :

$$
Y_{k n}=\mu+C_{k}+e_{k n}^{i}
$$

avec l'effet clone $\left(C_{k}\right)$ aléatoire. L'héritabilité clonale - sens large - a été calculée selon Nanson (1970) par :

$$
h^{2}{ }_{c}=\sigma_{d}^{2} /\left(\sigma_{c}^{2}+\sigma^{2} e^{1 / h_{o}}\right)
$$

d'après l'estimation des variances clone $\left(\sigma_{c}^{2}\right)$ et résiduelle $\left(\sigma_{e}^{2}\right)$ et le nombre moyen de ramets par clone $\left(n_{0}\right)$. Cette héritabilité clonale, déterminée d'après la valeur moyenne des clones, englobe également des effets génétiques liés aux niveaux descendance maternelle (additifs) et provenance. Elle permet d'évaluer la part des caractères qui est transmise lors de la multiplication par voie végétative d'une population multiclonale. L'écart type de cette héritabilité a été calculé selon Becker (1967).

La recherche des sources de variabilité génétique a été entreprise par la décomposition de la variance selon les différents niveaux d'après le modèle (3) :

$$
Y_{i j k n}=\mu+P_{i}+D_{j i j}+C_{k / i j}+e^{n}{ }_{n / j i k}
$$

avec les effets provenance $\left(P_{i}\right)$, descendance hiérarchisée dans les provenances $\left(D_{j i}\right)$ et clone hiérarchisé dans les descendances $\left(\mathrm{C}_{\mathrm{k}_{\text {ij }}}\right)$; tous ces facteurs ont été considérés comme aléatoires. Les provenances non structurées en descendances ont été écartées de l'analyse. Toutefois, les nombres de descendances par provenance (10 en moyenne), de clones par descendance ( 4 en moyenne) et de ramets par clone ( 5 en moyenne) sont à la fois très variables et très faibles. Dans ces conditions, il est évident que les analyses réalisées selon le modèle 3 n'apporteront qu'une valeur indicative sur la variance relative des différents niveaux génétiques.

\section{Analyse entre les sites}

Une analyse entre les clones et les sites a été effectuée, à partir des valeurs des ramets ajustées aux blocs, selon le modèle (4) :

$$
\mathrm{Y}_{\mathrm{kmn}}=\mu+\mathrm{C}_{\mathrm{k}}+\mathrm{S}_{\mathrm{m}}+\mathrm{e}^{\prime \prime \prime} \mathrm{kmn}
$$

avec les effets clone $\left(C_{k}\right.$, aléatoire) et site $\left(S_{m}\right.$, fixe). Le modèle n'est ni orthogonal ni équilibré. L'ajustement est réalisé d'après l'effet des sites $\left(Z_{k n}=Y_{k m n}-S_{m}\right)$. A ce stade, linteraction clone-site est négligée; elle sera étudiée ultérieurement.
Les paramètres génétiques des clones sur l'ensemble des sites sont estimés selon le modèle (5) à partir des valeurs individuelles ajustées aux effets bloc et site :

$$
Z_{k n}=\mu+C_{k}+e^{\prime \prime \prime}{ }_{k n}
$$

L'héritabilité est calculée selon la même méthode que pour le modèle 2.

Pour tirer le maximum d'informations de cette expérience, malgré l'absence de mesure de débourrement dans le site 1 , les analyses ont été réalisées sans débourrement puis avec celui-ci. Les premières permettent d'estimer les paramètres génétiques de la vigueur pour l'ensemble des clones et des sites, tandis que les dernières conduisent à une évaluation de l'héritabilité du débourrement et des corrélations entre ce caractère et la vigueur pour les clones implantés dans les sites 2 et 3 . Les corrélations phénotypiques ont été calculées à partir des moyennes clonales de la vigueur ( 1130 clones pour les 3 sites), ou de celles du débourrement (1 074 clones dans les sites 2 et 3 ).

Dans ce qui suit, le terme de variance est employé pour l'estimateur de la variance déterminé d'après l'espérance des carrés moyens. Les degrés de confiance sont notés : ns (non significatif à $5 \%$ ), * (significatif à $5 \%$ ), "* (significatil à $1 \%$ ) ou *** (significatif à $0,1 \%$ ).

\section{Résultats}

\section{Analyse intrasite}

Pour les 2 dispositifs, installés dans le Doubs, la sécheresse de 1983 a entraîné la mort de $12 \%$ des plants. En 1985, la mortalité moyenne pour les 3 dispositifs est respectivement de 21,26 et $2 \%$. L'effet bloc est très hautement significatif. Ainsi, la mortalité est plus élevée pour les blocs situés dans les parties hautes des dispositifs, zones où le sol est le moins profond. Dans l'ensemble, l'origine génétique a peu d'influence sur la mortalité.

D'une façon générale, l'effet bloc est toujours très hautement significatif. Pour la hauteur totale, il croît d'un facteur égal à 10 entre 1980 et 1985 . Quel que soit le caractère considéré, l'interaction clonesite n'est pas significative. 


\section{Hauteur totale}

L'évolution de la hauteur totale (Fig. 2) montre que les clones testés dans le site 1 présentent un avantage qui semble se maintenir. Cet avantage résulte de l'année supplémentaire qu'ont eu les plants du site 1 issus de la première campagne de bouturage. Pour les 2 autres dispositifs, d'âge comparable, la hauteur est plus faible dans le Massif central. Les 3 témoins sont plus petits que la moyenne générale des clones. Dans le site 1, Bonnétage dépasse Gérardmer, ce qui est inhabituel.

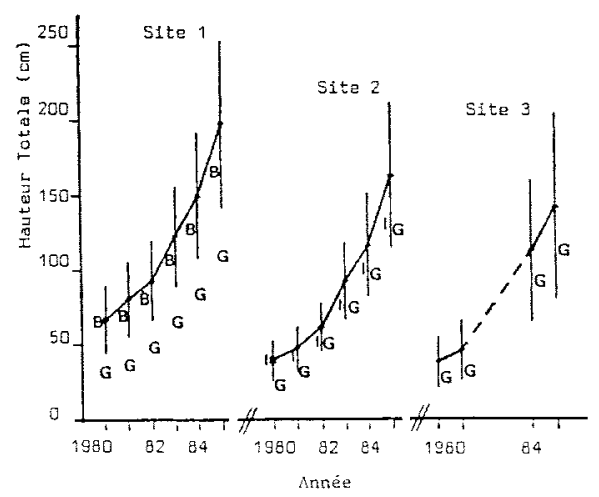

Fig. 2. Evolution de la hauteur totale calculee sur les moyennes de clones. La moyenne \pm l'écart type des clones est indiquée par une barre verticale. Bonnétage (B); Gerardmer (G); Istebna (I).

Les héritabilités clonales de la hauteur totale, calculées selon le modèle 2 , sont très fortes en sortie de pépinière (début de plantation). Ceci peut s'expliquer par de fortes variances entre clones traduisant des différences clonales pour l'aptitude au bouturage et la croissance en pépinière. En outre, la variance environnementale entre les différents ramets d'un même clone est faible par l'absence de dispositif statistique en pépinière lors du bouturage et du repiquage.
Les héritabilités clonales de la hauteur totale diminuent fortement entre 1980 et 1985, notamment pour le site 1 (Fig. 3). L'héritabilité semble amorcer une remontée en 1985 pour le site 2, alors qu'elle paraît proche du minimum pour le site 3 . Cette diminution résulte d'une augmentation de la variance résiduelle (crise de transplantation) et d'une diminution simultanée de la variance entre clones. La liaison entre la moyenne et la variance de la hauteur au cours du temps nécessiterait une transformation des valeurs dans le cas d'une étude comparative sur les sources de variation de l'héritabilité de ce caractère.

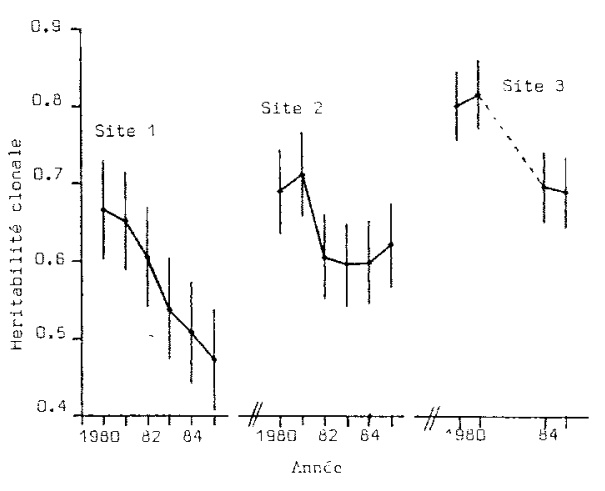

Fig. 3. Evolution de l'héritabilité clonale de la hauteur totale.

L'origine génétique de l'héritabilité de la hauteur totale peut être éclairée par l'analyse de la variance selon le modèle 3 . La variance résiduelle représente encore une part importante de la variance phénotypique totale (Tableau III). La variance totale liée aux différents niveaux génétiques varie de 17 à $28 \%$ selon le cas, et la variance clonale représente dans tous les cas près des deux tiers de ce total.

\section{Pousse annuelle}

La pousse annuelle (Fig. 4) augmente régulièrement au cours des années; la 
Tableau III. Analyse hiérarchique de la variance (modèle 2) pour la hauteur 1985.

\begin{tabular}{|c|c|c|c|c|c|c|c|c|c|}
\hline \multirow[t]{2}{*}{ Niveaux } & \multicolumn{3}{|c|}{ Site 1} & \multicolumn{3}{|c|}{ Site 2} & \multicolumn{3}{|c|}{ Site 3} \\
\hline & $d d l$ & $F$ & $\sigma^{2} \%$ & $d d l$ & $F$ & $\sigma^{2} \%$ & $d d l$ & $F$ & $\sigma^{2} \%$ \\
\hline $\begin{array}{l}\text { Provenance } \\
\text { Descendance/P. } \\
\text { Clone/Desc. } \\
\text { Résiduel }\end{array}$ & $\begin{array}{l}22 \\
205 \\
312 \\
2057\end{array}$ & $\begin{array}{l}2,2^{\star \star} \\
1,2^{*} \\
1,7^{* * *}\end{array}$ & $\begin{array}{l}2 \\
2 \\
13 \\
83\end{array}$ & $\begin{array}{l}23 \\
230 \\
454 \\
2611\end{array}$ & $\begin{array}{l}6,0^{\star \star \star} \\
1,3^{\star \star} \\
2,1^{\star \star \star}\end{array}$ & $\begin{array}{l}7 \\
3 \\
18 \\
72\end{array}$ & $\begin{array}{l}23 \\
267 \\
677 \\
4587\end{array}$ & $\begin{array}{l}5,0^{\star \star *} \\
1,2^{\star \star} \\
2,6^{\star \star *}\end{array}$ & $\begin{array}{l}4 \\
2 \\
20 \\
74\end{array}$ \\
\hline
\end{tabular}

Test $F$ de Fisher $(F)$. Part de la variance estimée en pourcentage de la variance phénotypique totale $\left(\sigma^{2} \%\right)$. Les clones sélectionnés en pépinière à partir de provenances ont été exclus de l'analyse. Les degrés de confiance sont notés ${ }^{*}$ significatif à $5 \%$; ${ }^{* *}$ significatif à $1 \% ;{ }^{* * *}$ significatif à $0,1 \%$.

vitesse de croissance n'a donc pas atteint son optimum. Pour les 2 dispositifs du Doubs (sites 1 et 2), la pousse annuelle est semblable et atteint $50 \mathrm{~cm}$ en 1985 . Pour le dispositif de la Creuse (site 3), la pousse n'est que de $30 \mathrm{~cm}$ en 1985. Là encore, les témoins ont une croissance plus lente que les clones.

L'héritabilité clonale (modèle 2) de la pousse annuelle est minimale en 19821983 pour les 2 premiers sites dont les mesures sont complètes (Fig. 5). La remontée de l'héritabilité de la pousse annuelle traduit l'expression d'autres aptitudes des clones vis-à-vis de la croissance à la suite des modifications de conditions environnementales rencontrées en

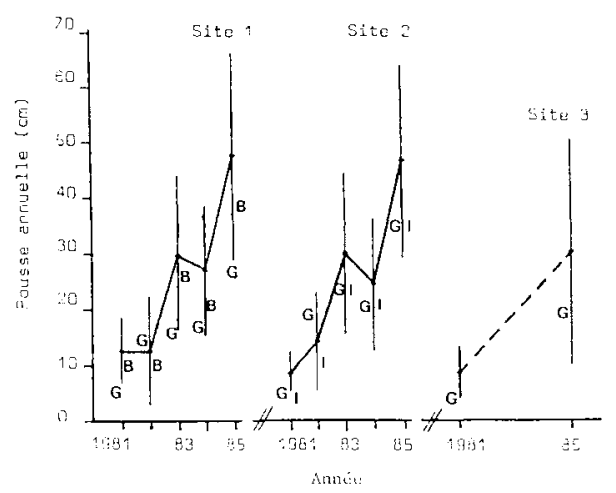

Fig. 4. Evolution de la pousse annuelle calculée sur les moyennes de clones. Même légende que pour la Figure 2. plantation. L'héritabilité de la pousse 1985 peut donc représenter une valeur minimale de l'héritabilité de la pousse annuelle à un âge plus avancé lorsque l'expression de ce caractère sera stabilisée. La décomposition de la variance selon les différents niveaux génétiques est comparable à celle indiquée sur le Tableau III pour la hauteur 1985.

\section{Pousse depuis la plantation}

La pousse cumulée de 1980 à 1985 est déterminée pour 5 ans de plantation, et peut donc être strictement comparée d'un site à l'autre. Elle présente les mêmes caractéristiques que la pousse annuelle et, comme pour la hauteur totale, les

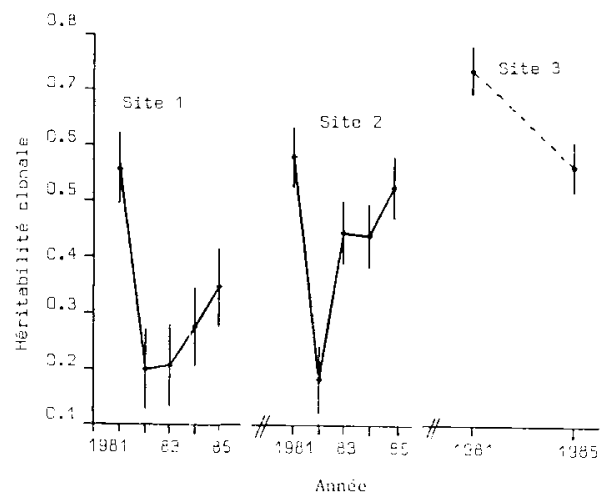

Fig. 5. Evolution de lihéritabilité clonale de la pousse annuelle. 
Tableau IV. Comparaison de la pousse cumulée de 1980 à 1985.

\begin{tabular}{llll}
\hline Moyenne $(\mathrm{cm})$ & Site 1 & Site 2 & Site 3 \\
\hline Clones & $129,5 \pm 43,3$ & $123,4 \pm 40,5$ & $105,2 \pm 54,1$ \\
Bonnétage & 104,7 & - & - \\
Gérardmer & 80,9 & 105,4 & 83,7 \\
Istebna & - & 97,3 & - \\
\hline
\end{tabular}

L'écart type phénotypique au seuil de $5 \%$ est indiqué pour les clones.

témoins ont des valeurs inférieures à la moyenne des clones (Tableau IV). Là encore, on remarque une supériorité surprenante de Bonnétage sur Gérardmer et de Gérardmer sur Istebna, ce qui diffère des résultats de Légnaté (1986). II est possible que cela traduise une meilleure adaptation de Bonnétage aux sols carbonatés du Jura.

Pour la pousse cumulée de 1980 à 1985 , les héritabilités clonales (modèle 2) sont respectivement de 0,32 , de 0,56 et de 0,63 pour les 3 sites. Elles sont intermédiaires entre celles de la hauteur totale et celles de la pousse annuelle 1985.

La décomposition de la variance (modèle 3) pour la pousse cumulée entre 1980 et 1985 souligne que la part prise par la variance résiduelle (Tableau $V$ ) est plus importante que celle indiquée précédemment pour la hauteur totale (Tableau III). Là encore, la variance attribuable aux 3 niveaux provenance, descendance et clone est particulièrement réduite pour le site 1.

\section{Débourrement végétatif}

Le débourrement n'a été mesuré que dans les dispositifs 2 et 3 . De ce fait, Bonnétage et 56 clones présents dans le seul site 1 n'ont pas pu être testés. Par rapport à la moyenne des clones, le témoin Gérardmer est plus précoce de 15 jours dans le site 2 ( 16 jours contre 31 jours en moyenne), de 12 jours dans le site 3 (4,5 jours contre 17 jours en moyenne). Le témoin Istebna (site 2) débourre 9 jours avant la moyenne des clones. Ces fortes différences de tardiveté expriment en partie le gain obtenu par les sélections faites sur ce caractère en forêt et en pépinière.

L'héritabilité clonale du débourrement (modèle 2) atteint 0,79 pour le site 2 et 0,89 pour le site 3 , ce qui confirme un contrôle génétique étroit.

L'analyse hiérarchique selon le modèle 3 montre que les effets sont tous très hautement significatifs et que les 3 niveaux génétiques expliquent de 52 à $57 \%$ de la variance totale (Tableau VI). La part de la

Tableau V. Analyse hiérarchique de la variance (modèle 3) pour la pousse cumulée de 1980 à 1985.

\begin{tabular}{|c|c|c|c|c|c|c|c|c|c|}
\hline \multirow[t]{2}{*}{ Niveaux } & \multicolumn{3}{|c|}{ Site 1} & \multicolumn{3}{|c|}{ Site 2} & \multicolumn{3}{|c|}{ Site 3} \\
\hline & $d d l$ & $F$ & $\sigma^{2} \%$ & $d d l$ & $F$ & $\sigma^{2} \%$ & $d d l$ & $F$ & $\sigma^{2} \%$ \\
\hline $\begin{array}{l}\text { Provenance } \\
\text { Descendance/P. } \\
\text { Clone/Desc. } \\
\text { Résiduel }\end{array}$ & $\begin{array}{l}22 \\
205 \\
312 \\
2057\end{array}$ & $\begin{array}{l}1,5 \mathrm{~ns} \\
1,2^{*} \\
1,5^{\star *}\end{array}$ & $\begin{array}{l}1 \\
2 \\
10 \\
87\end{array}$ & $\begin{array}{l}23 \\
230 \\
454 \\
2611\end{array}$ & $\begin{array}{l}5,2^{\star \star \star} \\
1,3^{\star *} \\
1,8^{\star * \star}\end{array}$ & $\begin{array}{l}5 \\
3 \\
14 \\
78\end{array}$ & $\begin{array}{l}23 \\
267 \\
677 \\
4587\end{array}$ & $\begin{array}{l}4,0 \text { ** } \\
1,2^{* *} \\
2,3^{* * *}\end{array}$ & $\begin{array}{l}* 3 \\
2 \\
17 \\
78\end{array}$ \\
\hline
\end{tabular}

Test $F$ de Fisher $(F)$. Part de la variance estimée en pourcentage de la variance phénotypique totale $\left(\sigma^{2} \%\right)$. Les clones sélectionnés en pépinère à partir de provenances ont été exclus de l'analyse. Degrés de confiance : voir Tableau III. 
Tableau VI. Analyse hiérarchique de la variance (modèle 3) pour le débourrement 1986.

\begin{tabular}{|c|c|c|c|c|c|c|}
\hline \multirow[t]{2}{*}{ Niveaux } & \multicolumn{3}{|c|}{ Site 2} & \multicolumn{3}{|c|}{ Site 3} \\
\hline & $d d l$ & $F$ & $\sigma^{2} \%$ & $d d l$ & $F$ & $\sigma^{2} \%$ \\
\hline Provenance & 23 & $6,8^{\star \star \star \star}$ & 13 & 23 & $10,7^{\star \star *}$ & 15 \\
\hline Descendance/P. & 230 & $1,7^{\star \star \star}$ & 9 & 267 & $1,5^{\star \star \star}$ & 6 \\
\hline Clone/Desc. & 454 & $3,9^{\star \star *}$ & 30 & 677 & $5,6^{\star \star *}$ & 36 \\
\hline Résiduel & 2611 & & 48 & 4587 & & 43 \\
\hline
\end{tabular}

Test $F$ de Fisher $(F)$. Part de la variance estimée en pourcentage de la variance phénotypique totale $\left(\sigma^{2} \%\right)$. Les clones sélectionnés en pépinère à partir de provenances ont été exclus de l'analyse. Degrés de confiance : voir Tableau III.

variance clonale dans la variance génétique des 3 niveaux intervient pour plus de la moitié, mais est légèrement moins importante que pour les caractères de vigueur.

\section{Corrélations entre caractères différents}

Les corrélations génétiques clonales (modèle 2) entre la hauteur 1985 et la pousse cumulée 1980-1985 sont très fortes et semblables selon les sites $(r=$ $0,92-0,98)$. En revanche, la corrélation génétique entre ces 2 caractères et le débourrement 1986 est négative pour le site 2 ( $r=-0,12$ pour 758 clones) et positive pour le site $3(r=0,28$ pour 1040 clones). Cette liaison faible est donc favorable ou défavorable à la sélection selon le site considéré. Cette différence de comportement pourrait s'expliquer par des compositions clonales différentes selon les sites.
Analyse inter-site

Corrélations entre les sites pour les mêmes caractères

Pour la hauteur 1985 (Tableau VII), les corrélations entre les moyennes des clones communs à 2 sites sont très hautement significatives mais n'expliquent que $18 \%$ de la variabilité dans le meilleur des cas. Pour la pousse cumulée de 1980 à 1985, les corrélations sont plus faibles et la part expliquée est réduite à $12 \%$, ce qui suggère que le classement des clones risque fort de varier d'un site à l'autre.

Pour le débourrement, la corrélation des 568 clones communs aux sites 2 et 3 est de 0,68 , et traduit une bonne stabilité des clones pour ce caractère.

\section{Performances moyennes}

L'ajustement des valeurs individuelles à l'effet moyen des sites a été effectué pour

Tableau VII. Corrélation entre les moyennes de clones communs à 2 sites.

\begin{tabular}{|c|c|c|c|}
\hline & & Site 2 & Site 3 \\
\hline \multirow[t]{2}{*}{ Hauteur totale (1985) } & site 1 & $0,31^{* * *}$ & $0 ; 35^{\star * *}$ \\
\hline & site 2 & - & $0,43^{\star \star \star *}$ \\
\hline \multirow[t]{2}{*}{ Pousse (1980-1985) } & site 1 & $0,26 * * *$ & $0,28^{* * *}$ \\
\hline & site 2 & - & $0,35^{\star \star \star *}$ \\
\hline Débourrement (1986) & site 2 & - & $0,68^{\star \star \star}$ \\
\hline
\end{tabular}

Degrés de confiance : voir Tableau ill. 
Tableau VIII. Caractéristiques moyennes des clones après ajustement à l'effet site.

Débourrement (1986) Hauteur totale (1985) Pousse cumulée (1980-1985)

\begin{tabular}{llll}
\hline Clones & & & \\
Etendue & $5,1-34,4 \mathrm{~J}$ & $92-245 \mathrm{~cm}$ & $55-181 \mathrm{~cm}$ \\
CV phénotyp. & $27,1 \%$ & $14,8 \%$ & $14,8 \%$ \\
Moyenne & $20,6 \mathrm{~J}$ & $155 \mathrm{~cm}$ & $110 \mathrm{~cm}$ \\
& & & \\
Témoins & $\mathrm{NM}$ & $128 \mathrm{~cm}$ & $83 \mathrm{~cm}$ \\
Bonnétage & $7,4 \mathrm{~J}$ & $109 \mathrm{~cm}$ & $85 \mathrm{~cm}$ \\
Gérardmer & $12,4 \mathrm{~J}$ & $130 \mathrm{~cm}$ & $85 \mathrm{~cm}$ \\
Istebna & 0,86 & 0,81 & 0,62 \\
Héritabilité clonale & 0,86 & \\
\hline
\end{tabular}
(NM).

les 3 caractères. La valeur extrême des clones varie d'un facteur de 3 pour la hauteur (Tableau VIII). Bien que cet écart paraisse élevé, le coefficient de variation phénotypique n'est que de $15 \%$. En outre, la performance des provenances témoins est très inférieure à la moyenne des clones. Ces 2 constatations soulignent l'efficacité des sélections entreprises en pépinière. Le gain obtenu sur le débourrement permet aux clones d'échapper à la plupart des gelées tardives, la valeur moyenne correspondant à un débourrement au 12 juin 1986.

L'héritabilité clonale de la pousse cumulée, bien qu'inférieure à celle de la hauteur totale, est encore très élevée. Elle approche la plus grande valeur des 3 estimations intrasites de l'héritabilité sur la hauteur totale.

Les corrélations, déterminées d'après les valeurs moyennes de 1074 clones, entre le débourrement 1986, d'une part, et la hauteur 1985 ou la pousse cumulée 1980-1985, d'autre part, sont toutes deux égales à 0,05 (ns). L'absence de liaison entre ces 2 types de caractères peut résulter d'une compensation entre les corrélations négative du site 2 et positive du site 3.

\section{Discussion et conclusion}

Cette analyse, malgré le stade juvénile des tests clonaux, met en évidence des résultats cohérents d'un site à l'autre. Ceci se remarque aussi bien pour la décomposition de la variance selon les différents niveaux génétiques que pour les estimations d'héritabilités.

La différence d'évolution au cours du temps entre l'héritabilité clonale de la hauteur et celle de la pousse en plantation (1980-1985) suggère 2 hypothèses. La crise de transplantation, par les bouleversements physiologiques qu'elle provoque, peut perturber provisoirement l'expression de gènes qui contrôlent la croissance. L'héritabilité pour la hauteur devrait alors revenir progressivement à un niveau proche de 0,70 , valeur observée juste après la plantation. II est également possible que des gènes s'expriment dans les bonnes conditions de la pépinière et que d'autres prennent le relais ou s'associent en conditions plus difficiles. Ce passage d'un jeu de gènes à un autre pourrait expliquer d'une part, la chute brutale de l'héritabilité, également constatée par Roulund et al. (1985), et, d'autre part, les 
faibles corrélations entre ortets et ramets observées par Birot et Nepveu (1979), Roulund et al. (1986) et Skroppa et Dietrichson (1986). L'étude des corrélations pépinière-terrain sur les clones étudiés ici fera l'objet d'un autre article.

Du point de vue pratique, l'héritabilité de la hauteur totale n'a peut-être pas encore atteint son minimum après 5 années de plantation, alors que l'héritabilité de la pousse annuelle est croissante depuis la troisième année de plantation. L'estimation de l'héritabilité pour la vigueur risque d'être surestimée si l'on utilise la première, et sous-estimée si l'on utilise la seconde. La pousse cumulée en plantation correspond donc à une valeur minimale espérée et peut être considérée à ce stade comme le caractère le plus fiable sur lequel pourra porter une sélection sur la vigueur.

Un autre point intéressant est la faiblesse des corrélations au niveau des moyennes de clones entre la vigueur et le débourrement, ou même l'absence de liaison lorsque les 3 sites sont étudiés globalement. Bien que des corrélations négatives entre ces 2 caractères aient été plusieurs fois constatées (Kleinschmit et Sauer, 1976; Birot et Nepveu, 1979; Lacaze et Kociecki, 1979), certaines études tendent à prouver que le sens de la liaison entre croissance et tardiveté varie chez l'épicéa commun selon les provenances étudiées (Birot et al., 1976; Légnaté, 1986). Les corrélations faibles ou nulles constatées dans cette expérience pourraient alors résulter du nombre important de provenances originaires de diverses régions dans lesquelles les clones ont été retenus : les corrélations positives des unes compensant les corrélations négatives des autres. Sous cette hypothèse, la différence de composition clonale d'un site à l'autre expliquerait en grande partie l'apparition de corrélations génétiques positives dans un site et négatives dans un autre, d'ailleurs de faible valeur absolue.
Dans l'ensemble, on pourra donc envisager de conserver des clones à la fois vigoureux et tardifs.

Les clones étudiés sont le résultat d'une sélection phénotypique en forêt et d'une forte sélection des ortets en pépinière. II est donc nécessaire d'estimer l'impact de ces sélections sur les performances et certaines caractéristiques des clones.

L'influence de la sélection phénotypique réalisée en forêt (arbres «+»), sur la tardiveté du débourrement et la densité du bois, est très hautement significative. Les clones issus d'arbres "+» débourrent en moyenne 2,6 jours après ceux d'arbres tout-venant. A titre d'exemple, pour Istebna, les clones issus d'arbres «+» (ISTE «+») sont plus tardifs de 2,2 jours que ceux des peuplements correspondants (IS-BU et IS-ZA). En revanche, pour la hauteur et la pousse cumulée, on ne constate pas de différence entre les populations clonales issues d'arbres «+» et d'arbres tout-venant. II paraît donc que, dans ce cas, la sélection phénotypique en forêt n'a pas entraîné de contre-sélection sur la vigueur, et cela, malgré les corrélations négatives fréquemment observées entre la vigueur et la densité du bois (Lacaze et Polge, 1970; Birot et Nepveu, 1979; Lewark, 1979) ou le débourrement par ces 2 premiers auteurs et Birot et al. (1976), Kleinschmit et Sauer (1976) puis Légnaté (1986).

L'influence de la sélection en pépinière sur les performances peut s'estimer par la comparaison de la moyenne des clones et de la meilleure performance des 3 provenances témoins. Bien que les tests clonaux ne renterment pas de témoin strictement représentatif, le gain réalisé semble élevé puisque les clones sont plus vigoureux de $25 \mathrm{~cm}$, ce qui correspond à une année supplémentaire de végétation pour les témoins, et plus tardifs de 8,2 jours, ce qui les met pratiquement à l'abri des gelées tardives. De même pour le débour- 
rement, on constate un gain moyen de 7 jours pour les clones issus des provenances IS-BU et IS-ZA comparés aux semis témoins d'Istebna. De la sorte, la sélection en forêt (+2,2 jours) et celle en pépinière ( +7 jours) apportent un gain global de 9 jours pour le matériel originaire de la région d'Istebna.

La sélection en pépinière a-t-elle modifié les caractéristiques moyennes des populations ? L'expérience clonale présentée ici ne permet pas de répondre en toute rigueur. Toutefois, l'influence de l'origine géographique des clones est encore très marquée pour le matériel polonais. Ainsi, en moyenne, les clones issus des provenances du nord-est (Baltique) sont très tardifs (débourrement de 22,2 jours pour une hauteur de $149 \mathrm{~cm}$ ) alors que ceux de la zone sud (Beskides) sont très vigoureux (hauteur de $160 \mathrm{~cm}$ pour un débourrement de 19,8 jours). Les clones du centre de la Pologne paraissent à la fois plus vigoureux (hauteur de $166 \mathrm{~cm}$ ) et plus tardifs (23 jours) que ceux des 2 zones précédentes, bien que ces différences ne soient pas significatives. Les caractères distinctifs des provenances polonaises, tels que décrits notamment par Lacaze et Kociecki (1979) puis par Légnaté (1986), sont donc encore nettement visibles à travers les clones qui en sont issus.

L'impact de la sélection précoce sur la variabilité génétique des clones doit être abordée avec prudence, car la représentation des divers niveaux génétiques est très déséquilibrée. De plus, les faibles effectifs limitent la précision des estimations des variances liées aux nivaux provenance et descendance, et deviennent insuffisants pour le niveau clonal (Russel et Libby, 1986). On constate cependant que la proportion de variance cumulée pour les niveaux provenance et descendance est comprise entre 4 et $10 \%$ pour la hauteur et entre 21 et $22 \%$ pour le débourrement.
Ces valeurs sont légèrement plus faibles que celles déterminées par Légnaté (1986) sur des semis du même matériel, qui étaient 10 à $20 \%$ pour la hauteur et 18 à $40 \%$ pour le débourrement, ou par Skroppa et Dietrichson (1986). La sélection en pépinière a donc entraîné une perte de variabilité. Toutefois, le mode de sélection utilisé, qui a permis de conserver toutes les provenances et les trois quarts des descendances initiales alors que 1,5\% de plants seulement étaient retenus comme ortets, a limité cette perte de variabilité.

Aux niveaux provenance et descendance, vient s'ajouter la variabilité clonale. En effet, le clonage a permis de fixer, sous forme de variance clonale, une partie de l'énorme variabilité individuelle (Skroppa et Dietrichson, 1986). La variance fixée est partois 3 tois plus importante que celle des niveaux provenance et descendance cumulés. En conséquence, les valeurs de I'héritabilité clonale sont élevées et du même ordre de grandeur que celles indiquées par Kleinschmit et Sauer (1976), Birot et Nepveu (1979), Roulund et al. $(1985,1986)$, St Clair et Kleinschmit (1986) et Skroppa et Dietrichson (1986).

Les résultats présentés pour ces 1130 clones permettent une première évaluation de la stratégie mise en cuvre par I'INRA pour la création de variétés multiclonales. Le matériel de départ était constitué par une population très large de provenances et de descendances ayant déjà montré leur valeur en test. Ainsi, avant toute création clonale, une grande variabilité génétique et un bon niveau moyen de performances étaient assurés. La sélection en pépinière, qui est à la base de cette création clonale, a été réalisée avec une forte intensité sur le caractère de débourrement : ce dernier ayant une héritabilité très élevée et une excellente stabilité dans l'espace et dans le temps, un gain génétique important se manifeste en 
forêt. En outre, la population clonale constituée est plus vigoureuse en moyenne que les témoins et renferme une très forte variabilité pour les caractères de croissance. Cette dernière propriété, liée aux modifications des paramètres génétiques entre la pépinière et le terrain, justifie a posteriori l'option qui a été prise de réaliser la principale sélection sur la croissance en forêt.

Ainsi, les 1130 clones étudiés ici présentent :

- un niveau de performance nettement amélioré sur 2 caractères économiquement importants;

- une proportion élevée de la variabilité génétique des populations dont ils sont issus et de fortes héritabilités clonales;

- une absence de corrélation défavorable entre croissance et tardiveté.

Toutes ces caractéristiques permettent d'espérer à la fois le maintien d'une base génétique large et l'obtention de nouveaux gains génétiques importants lors des prochaines étapes de sélection des clones. Ces dernières porteront prioritairement sur les caractères de croissance et de qualité du bois, ainsi que sur la plasticité des clones.

\section{Références}

Becker W.A. (1967) Manual of Procedures in Quantitative Genetic. Washington State University Press, $2^{\boldsymbol{\theta}}$ éd.

Birot Y., Lacaze J.F. \& Le Couviour J. (1976) About the reliability of mass selection in forest : an example in Norway spruce (Picea abies Karst.). IUFRO Joint Meeting of Advanced Generation Breeding, Bordeaux, 14-18 juin

Birot Y. \& Nepveu G. (1979) Variabilité clonale et liaisons ortets-ramets dans une population d'épicéa. Silvae Genet. 28 (2-3), 37-47

Burdon R.D. (1986) Clonal forestry and breeding strategies. A perspective. IUFRO Confe- rence, Williamsburg (Virginia, Etats-Unis), Proc. Conf., pp. 645-659

Huehn M., Kleinschmit J. \& Svolba J. (1987) Some experimental results concerning age dependency of different components of variance in testing Norway spruce (Picea abies (L.) Karst.) clones. Silvae Genet. 36 (2), 68-71

Kleinschmit J. (1983) Concepts and experiences in clonal plantations of conifers. Canadian Tree Improvement Association, 19th Biennial Meeting, Toronto, 23-26 août

Kleinschmit J. \& Sauer A. (1976) Variation in morphology, phenology and nutrient content among Picea abies clones and provenances and its implications for tree improvement. In : Tree Physiclogy and Yield Improvement, (M.G.R. Cannell \& F.T. Last, éds.), Academic Press, New York, pp. 503-517

Lacaze J.F. \& Polge H. (1970) Relations phénotypiques au stade juvénile entre la densité du bois et divers caractères phénologiques et de vigueur chez Picea abies Karst. Ann. Sci. For. $27,231-242$

Lacaze J.F. \& Kociecki S. (1979) Zmiennosé populacji polskiego swierka na powierzchniach we Francji w Polsce. (Variabilité de populations polonaises d'épicéa en France et en Pologne). Sylwan 6, 1-21

Légnaté N.H. (1986) Etude de la structure génétique des populations polonaises d'épicéa commun (Picea abies Karst.). Corrélations génétiques. Héritabilité. Espérance de gains génétiques. DEAS Univ. Orsay Paris-Sud, Rapport interne INRA

Lewark S. (1979) Wood characteristics in Norway spruce breeding programs. IUFRO Norway Spruce Meeting, Bucharest, pp. 316-341

Matheson A.C. \& Lindgren D. (1985) Gains from the clonal and the clonal seed-orchard options compared for tree breeding programs. Theor. Appl. Genet. 71, 242-249

Monchaux P. (1984) De l'interaction clone $x$ environnement chez l'épicéa commun. AFOCEL, Rapport annuel, pp. 247-275

Nanson A. (1970) L'héritabilité et le gain d'origine génétique dans quelques types d'expériences. Silvae Genet. 19 (4), 113-121

Roulund $H$., Wellendorf $H$. \& Werner M. (1985) A clonal experiment in Norway spruce (Picea abies (L.) Karst.) : 15 years' results. For. Tree Improv. 17, 1-33

Roulund H., Wellendorf H. \& Werner M. (1986) A selection experiment for height growth with 
cuttings of Picea abies (L.) Karst. Scand. J. For. Res. 1, 293-302

Russel J.H. \& Libby W.J. (1986) Clonal testing efficiency : the trade-offs between clones tested and ramets per clone. J. For. Res. 16, 925930

St Clair J.B. \& Kleinschmit J. (1986) Genotypeenvironment interaction and stability in tenyear height growth of Norway spruce clones
(Picea abies Karst.). Silvae Genet. 35 (5-6), 177-186

St Clair J.B., Kleinschmit J. \& Svolba J. (1985) Juvenility and serial vegetative propagation of Norway spruce clones (Picea abies Karst.). Silvae Genet. 34 (1), $42-48$

Skroppa T. \& Dietrichson J. (1986) Genetic variation and ortet-ramet relationships in a clonal test with Picea abies. Scand J. For. Res. 1, 323-332 